\title{
Embedding Cohen algebras using pcf theory
}

\author{
by
}

Saharon Shelah (Jerusalem and New Brunswick, NJ)

\begin{abstract}
Using a theorem from pcf theory, we show that for any singular cardinal $\nu$, the product of the Cohen forcing notions on $\kappa, \kappa<\nu$, adds a generic for the Cohen forcing notion on $\nu^{+}$.
\end{abstract}

The following question (problem 5.1 in Miller's list [Mi91]) is attributed to René David and Sy Friedman:

Does the product of the forcing notions $\aleph_{n}>2$ add a generic for the forcing $\aleph_{\omega+1}>2 ?$

We show here that the answer is yes in ZFC. Previously Zapletal [Za] showed this result under the assumption $\square_{\aleph_{\omega+1}}$.

In fact, a similar theorem can be shown about other singular cardinals as well. The reader who is interested only in the original problem should $\operatorname{read} \aleph_{\omega+1}$ for $\lambda, \aleph_{\omega}$ for $\mu$ and $\left\{\aleph_{n}: n \in(1, \omega)\right\}$ for $\mathfrak{a}$.

We thank Martin Goldstern for writing up this article.

Definition 1. (1) Let $\mathfrak{a}$ be a set of regular cardinals. $\prod \mathfrak{a}$ is the set of all functions $f$ with domain $\mathfrak{a}$ satisfying $f(\kappa) \in \kappa$ for all $\kappa \in \mathfrak{a}$.

(2) A set $\mathfrak{b} \subseteq \mathfrak{a}$ is bounded if $\sup \mathfrak{b}<\sup \mathfrak{a}$, and cobounded if $\mathfrak{a} \backslash \mathfrak{b}$ is bounded.

(3) If $J$ is an ideal on $\mathfrak{a}, f, g \in \prod \mathfrak{a}$, then $f<_{J} g$ means $\{\kappa \in \mathfrak{a}: f(\kappa) \nless$ $g(\kappa)\} \in J$. We write $\prod \mathfrak{a} / J$ for the partial (quasi)order $\left(\prod \mathfrak{a},<_{J}\right)$.

(4) $\lambda=\operatorname{tcf}\left(\prod \mathfrak{a} / J\right)\left(\lambda\right.$ is the true cofinality of $\left.\prod \mathfrak{a} / J\right)$ means that there is a strictly increasing cofinal sequence of functions in the partial order $\left(\prod \mathfrak{a},<_{J}\right)$.

(5) $\operatorname{pcf}(\mathfrak{a})=\left\{\lambda:(\exists J)\left(\lambda=\operatorname{tcf}\left(\prod \mathfrak{a} / J\right)\right)\right\}$

2000 Mathematics Subject Classification: 03E40, 03E04.

Key words and phrases: set theory, pcf, forcing.

Research partially supported by "The Israel Science Foundation" administered by The Israel Academy of Sciences and Humanities. Publication 595. 
We will use the following theorem from pcf theory:

LEMMA 2. Let $\mu$ be a singular cardinal. Then there is a set $\mathfrak{a}$ of regular cardinals below $\mu$ with $|\mathfrak{a}|=\operatorname{cf}(\mu)<\min \mathfrak{a}$ and $\mu^{+} \in \operatorname{pcf}(\mathfrak{a})$. Moreover, we can even have $\operatorname{tcf}\left(\prod \mathfrak{a} / J^{\mathrm{bd}}\right)=\mu^{+}$, where $J^{\mathrm{bd}}$ is the ideal of all bounded subsets of $\mathfrak{a}$.

Proof. See [Sh 355, Theorem 1.5].

TheOREM 3. Let $\mathfrak{a}$ be a set of regular cardinals, $\mu=\sup \mathfrak{a} \notin \mathfrak{a}, 2^{<\lambda}=2^{\mu}$, $\lambda>\mu, \lambda \in \operatorname{pcf}(\mathfrak{a})$, and moreover:

(*) There is an ideal $J$ on $\mathfrak{a}$ containing all bounded sets such that $\lambda=$ $\operatorname{tcf}\left(\prod \mathfrak{a} / J\right)$.

Then the forcing notion $\prod_{\kappa \in \mathfrak{a}}{ }^{\kappa>} 2$ adds a generic for ${ }^{\lambda>} 2$.

COROLlary 4. If $\nu$ is a singular cardinal, and $P$ is the product of the forcing notions ${ }^{\kappa>} 2$ for $\kappa<\nu$, then $P$ adds a generic for ${ }^{\nu^{+}}>2$.

Proof. By Lemma 2 and Theorem 3.

REMARK 5. (1) The condition (*) in the theorem is equivalent to:

$(* *) \quad$ For all bounded sets $\mathfrak{b} \subset \mathfrak{a}$ we have $\lambda \in \operatorname{pcf}(\mathfrak{a} \backslash \mathfrak{b})$.

(2) Clearly the assumption $2^{<\lambda}=2^{\mu}$ is necessary, because otherwise the forcing notion $\prod_{\kappa \in \mathfrak{a}}{ }^{\kappa>}>2$ would be too small to add a generic for ${ }^{\lambda>} 2$.

Proof of Theorem 3. By our assumption we have some ideal $J$ containing all bounded sets such that $\operatorname{tcf}\left(\prod \mathfrak{a} / J\right)=\lambda$.

We will write $\left(\forall^{J} \kappa \in \mathfrak{a}\right)(\varphi(\kappa))$ for $\{\kappa \in \mathfrak{a}: \neg \varphi(\kappa)\} \in J$. So we have a sequence $\left\langle f_{\alpha}: \alpha<\lambda\right\rangle$ such that:

(a) $f_{\alpha} \in \prod \mathfrak{a}$.

(b) If $\alpha<\beta$, then $\left(\forall^{J} \kappa \in \mathfrak{a}\right)\left(f_{\alpha}(\kappa)<f_{\beta}(\kappa)\right)$.

(c) $\left(\forall f \in \prod \mathfrak{a}\right)(\exists \alpha)\left(\forall^{J} \kappa \in \mathfrak{a}\right)\left(f(\kappa)<f_{\alpha}(\kappa)\right)$.

The next lemma shows that if we allow these functions to be defined only almost everywhere, then we can additionally assume that in each block of length $\mu$ these functions have disjoint graphs:

Lemma 6. Assume that $\mathfrak{a}, \lambda, \mu$ are as above. Then there is a sequence $\left\langle g_{\alpha}: \alpha<\lambda\right\rangle$ such that:

(a) $\operatorname{dom}\left(g_{\alpha}\right) \subseteq \mathfrak{a}$ is cobounded (so in particular $\left(\forall^{J} \kappa \in \mathfrak{a}\right)(\kappa \in$ $\left.\operatorname{dom}\left(g_{\alpha}(\kappa)\right)\right)$.

(b) If $\alpha<\beta$, then $\left(\forall^{J} \kappa \in \mathfrak{a}\right)\left(g_{\alpha}(\kappa)<g_{\beta}(\kappa)\right)$.

(c) $\left(\forall f \in \prod \mathfrak{a}\right)(\exists \alpha)\left(\forall^{J} \kappa \in \mathfrak{a}\right)\left(f(\kappa)<g_{\alpha}(\kappa)\right)$. Moreover, we may choose $\alpha$ to be divisible by $\mu$.

(d) If $\alpha<\beta<\alpha+\mu$, then $\left(\forall \kappa \in \operatorname{dom}\left(g_{\alpha}\right) \cap \operatorname{dom}\left(g_{\beta}\right)\right)\left(g_{\alpha}(\kappa)<g_{\beta}(\kappa)\right)$. 
Proof. Let $\left\langle f_{\alpha}: \alpha<\lambda\right\rangle$ be as above. Now define $\left\langle g_{\alpha}: \alpha<\lambda\right\rangle$ by induction as follows:

If $\alpha=\mu \cdot \zeta$, then let $g_{\alpha} \in \prod \mathfrak{a}$ be any function that satisfies $g_{\beta}<_{J} g_{\alpha}$ for all $\beta<\alpha$, and also $f_{\alpha}<_{J} g_{\alpha}$. Such a function can be found because the set of functions of size $<\lambda$ can be $<_{J}$-bounded by some $f_{\beta}$.

If $\alpha=\mu \cdot \zeta+i, 0<i<\mu$, then let

$$
g_{\alpha}(\kappa)= \begin{cases}g_{\mu \cdot \zeta}(\kappa)+i & \text { if } i<\kappa \\ \text { undefined } & \text { otherwise. }\end{cases}
$$

It is easy to see that (a)-(d) are satisfied.

Definition 7. (1) Let $P_{\kappa}$ be the set ${ }^{\kappa>} 2$, partially ordered by inclusion (= sequence extension). Let $P=\prod_{\kappa \in \mathfrak{a}} P_{\kappa}$. [We will show that $P$ adds a generic for ${ }^{\lambda>2}$.]

(2) Assume that $\left\langle g_{\alpha}: \alpha<\lambda\right\rangle$ is as in Lemma 6 .

(3) Let $H:{ }^{\mu} 2 \rightarrow^{\lambda>} 2$ be onto.

(4) For $\kappa \in \mathfrak{a}$, let $\eta_{\kappa}$ be the $P_{\kappa}$-name for the generic function from $\kappa$ to 2 . Define a $P$-name of a function $\underset{\sim}{h}: \lambda \rightarrow 2$ by

$$
\underset{\sim}{h}(\alpha)= \begin{cases}0 & \text { if }\left(\forall^{J} \kappa \in \mathfrak{a}\right)\left(\eta_{\kappa}\left(g_{\alpha}(\kappa)\right)=0\right), \\ 1 & \text { otherwise. }\end{cases}
$$

(5) For $\xi<\lambda$ let $\varrho_{\xi}$ be a $P$-name for the element of $\mu_{2}$ that satisfies $\varrho_{\xi} \simeq \underset{\sim}{h}\lceil[\mu \cdot \xi, \mu \cdot(\xi+1))$, i.e.,

$$
i<\mu \Rightarrow \Vdash_{P} \varrho_{\mathcal{\xi}}(i)=\underset{\sim}{h}(\mu \cdot \xi+i) .
$$

Define $\underset{\sim}{\varrho} \in{ }^{\lambda} 2$ by

$$
\varrho=H\left(\underline{\varrho}_{0}\right) \frown H\left(\underline{\varrho}_{1}\right) \frown \ldots \frown H\left(\underline{\varrho}_{\xi}\right) \frown \ldots
$$

Main Claim 8. $\varrho$ is generic for ${ }^{\lambda>} 2$.

Definition 9. For $\alpha<\lambda$ let $P^{(\alpha)}$ be the set of all conditions $p$ satisfying $\left(\forall^{J} \kappa\right)\left(\operatorname{dom}\left(p_{\kappa}\right)=g_{\alpha}(\kappa)\right)$.

Remark 10. $\bigcup_{\zeta<\lambda} P^{(\mu \cdot \zeta)}$ is dense in $P$.

Proof. By Lemma 6(c).

FACT 11. Let $\alpha=\mu \cdot \zeta, p \in P^{(\alpha)}, \sigma \in{ }^{\mu} 2$. Then there is a condition $q \in P^{(\alpha+\mu)}, q \geq p$ and

$$
(\forall j<\mu)\left(\forall^{J} \kappa\right)\left(q_{\kappa}\left(g_{\alpha+j}(\kappa)\right)=\sigma(j)\right) .
$$

Proof. Let $p=\left(p_{\kappa}: \kappa \in \mathfrak{a}\right)$. There is a set $\mathfrak{b} \in J$ such that for all $\kappa \in \mathfrak{a} \backslash \mathfrak{b}$ we have $\operatorname{dom}\left(p_{\kappa}\right)=g_{\alpha}(\kappa)$. Define $q \in P^{(\alpha+\mu)}, q=\left(q_{\kappa}: \kappa \in \mathfrak{a}\right)$, as follows:

$$
q_{\kappa}(\gamma)= \begin{cases}p_{\kappa}(\gamma) & \text { if } \gamma \in \operatorname{dom}\left(p_{\kappa}\right) \\ \sigma(j) & \text { if } \gamma=g_{\alpha+j}(\kappa), \kappa \in \mathfrak{a} \backslash \mathfrak{b} \\ 0 & \text { otherwise }\end{cases}
$$


We have to explain why $q$ is well defined: First note that the first and the second case are mutually exclusive. Indeed, if $\gamma=g_{\alpha+j}(\kappa)$, then $\gamma>g_{\alpha}(\kappa)$, whereas $\kappa \notin \mathfrak{b}$ implies that $\operatorname{dom}\left(p_{\kappa}\right)=g_{\alpha}(\kappa)$, so $\gamma \notin \operatorname{dom}\left(p_{\kappa}\right)$.

Next, by the property (d) from Lemma 6 there is no contradiction between various instances of the second case. Also the third case causes no contradiction. Now obviously $q_{\kappa} \in P_{\kappa}$ and $p_{\kappa} \leq q_{\kappa}$. So $p \leq q \in P_{\kappa}$.

Hence we find that for all $j<\mu$, whenever $\kappa \in \mathfrak{a} \backslash \mathfrak{b}$ and $\kappa>j$, then $q_{\kappa}\left(g_{\alpha+j}(\kappa)\right)=\sigma(j)$. Since $J$ contains all bounded sets, this means that $\left(\forall^{J} \kappa\right)\left(q_{\kappa}\left(g_{\alpha+j}(\kappa)\right)=\sigma(j)\right)$.

Remark 12. Assume that $\alpha=\mu \cdot \zeta$, and $p, q, \sigma$ are as above. Then $q \Vdash \varrho_{\zeta}=\sigma$.

Proof of the main claim. Let $p \in P$, and $D \subseteq{ }^{\lambda>} 2$ be a dense open set. We may assume that for some $\alpha^{*}<\lambda$, $\zeta^{*}<\lambda$ we have $\alpha^{*}=\mu \cdot \zeta^{*}$ and $p \in P^{\left(\alpha^{*}\right)}$, i.e., for some $\mathfrak{b} \in J$ we have $(\forall \kappa \notin \mathfrak{b})\left(\operatorname{dom}\left(p_{\kappa}\right)=g_{\alpha^{*}}(\kappa)\right)$, by Remark 10.

So $p$ decides the values of $h \uparrow \alpha^{*}$, and hence also the values of $\varrho_{\zeta}$ for $\zeta<\zeta^{*}$. Specifically, for each $\zeta<\zeta^{*}$ we can define $\sigma_{\zeta} \in{ }^{\mu} 2$ by

$$
\sigma_{\zeta}(i)= \begin{cases}0 & \text { if }\left(\forall^{J} \kappa\right)\left(p_{\kappa}\left(g_{\mu \cdot \zeta+i}(\kappa)\right)=0\right) \\ 1 & \text { otherwise. }\end{cases}
$$

(Note that for all $\zeta<\zeta^{*}$ and all $i<\mu$, and almost all $\kappa$ the value of $p_{\kappa}\left(g_{\mu \cdot \zeta+i}(\kappa)\right)$ is defined.)

Clearly $p \Vdash \varrho_{\zeta}=\sigma_{\zeta}$. Since $D$ is dense and $H$ is onto, we can now find $\sigma_{\zeta^{*}} \in{ }^{\mu} 2$ such that $H\left(\sigma_{0}\right) \frown \ldots \frown H\left(\sigma_{\zeta}^{*}\right) \in D$. Using 11 and 12 , we can now find $q \geq p$ such that $q \Vdash \varrho_{\zeta^{*}}=\sigma_{\zeta^{*}}$.

Hence $q \Vdash \underset{\sim}{\varrho} \in D$, and we are done.

\section{References}

[Mi91] A. Miller, Arnie Miller's problem list, in: H. Judah (ed.), Set Theory of the Reals, Israel Math. Conf. Proc. 6, Bar-Ilan Univ., Ramat Gan, 1993, 645-654.

[Za] J. Zapletal, Some results in set theory and Boolean algebras, PhD thesis, Penn State Univ., 1995.

[Sh 355] S. Shelah, $\aleph_{\omega+1}$ has a Jonsson algebra, in: Cardinal Arithmetic, Oxford Logic Guides, Chapter II, Oxford Univ. Press, 1994.

Institute of Mathematics

The Hebrew University of Jerusalem 91904 Jerusalem, Israel

E-mail: shelah@math.huji.ac.il
Department of Mathematics Rutgers University New Brunswick, NJ 08854, U.S.A. 